Employment and Caring in British and Norwegian banking: an exploration through individual careers

Published in Work, Employment \& Society (2000) Vol. 14, No. 2: 331-352

Rosemary Crompton, City University London and

Gunn Elisabeth Birkelund, University of Oslo g.e.birkelund@sosiologi.uio.no

Department of Sociology and Human Geography University of Oslo

P.O.Box 1096 Blindern

N-0317 OSLO Norway

Telephone: $\quad+4722855257$

Fax: $\quad+4722855253$

Internet: http://www.iss.uio.no 


\title{
Employment and Caring in British and Norwegian banking: an exploration through individual careers
}

\section{Rosemary Crompton and Gunn Elisabeth Birkelund}

City University London

University of Oslo

\begin{abstract}
The continuing expansion of women's employment has increasingly focused attention on the question of how the caring work traditionally carried out by unpaid women will be accomplished. In particular, how can caring responsibilities be combined with a long-term career? In this paper, we assess the significance of the national context through a comparison of the biographies of career bank managers, male and female, in Britain and Norway. We find that although the (considerably) more family-friendly policies of the Norwegian state do have a positive impact, particularly in somewhat exceptional cases, nevertheless in general in both countries, individuals have difficulties in combining employment with caring and no examples of a 'two career' household were found in either country. These findings emphasise the continuing tension between market forces and social reproduction, which has been exacerbated by the erosion of the 'male breadwinner' model of family caring and has yet to be resolved in a satisfactory manner.
\end{abstract}

\section{Introduction}

In all European countries, it is increasingly recognised that it can no longer be assumed that major caring responsibilities, particularly childcare, may be safely left to non-employed wives. The decline of the 'male breadwinner' model of family care, together with the rise in dual earner households, is recognised in European policy statements, and a national childcare policy is being developed even in countries such as Britain, which has long been acknowledged to have one of the lowest levels of publicly provided childcare (Melhuish and Moss 1991).

However, at the same time as these changes are taking place in attitudes to families and family policy, it would seem that other pressures are making the world of paid employment increasingly family unfriendly - especially for those wanting to develop successful careers (Hochschild 1997). Indeed, a recent British study has suggested that although women are no longer the focus of direct exclusionary practices within the workplace, in career terms, a new division is opening up within organisations; between 'encumbered' and 'unencumbered' workers - that is, those

Rosemary Crompton is in the Department of Sociology School of Social \& Human Sciences at the City University, London. Gunn Elisabeth Birkelund is in the Department of Sociology at the University of Oslo, Norway. 
with and without caring responsibilities (Halford et al. 1997). Does this mean that current exhortations to employers (for example, by the British Government) to enhance the extent of their 'family friendliness' will prove to be, in the last analysis, relatively meaningless?

It is not easy to give a simple answer to this rhetorical question. However, in this paper we will be discussing the insights that can be gained from a comparative study of career managers in retail banking in two countries with very different national approaches to employment and caring - Britain and Norway. As a Scandinavian welfare state, Norway is characterised by 'family friendly' national employment policies that have recently been extended to include 'political fatherhood' (Ellingsaeter 1999). ${ }^{1}$ In contrast, the British state has not, until very recently, sought to give any help to working mothers, and in welfare terms, would be classified as 'liberal' (Esping-Andersen 1990), offering very little by way of assistance with care. To what extent are the more 'family-friendly' policies of the Norwegian state actually reflected in individual careers?

\section{Gender and Retail Banking in Britain and Norway}

In both Britain and Norway, retail banking was once the preserve of the masculine bureaucratic career. Managers were long-term employees who had worked their way through the internal labour market, and aspiring young men were recruited into a 'job for life' (Crompton and Jones 1984). The British clearing banks ${ }^{2}$ prided themselves on training all their professional staff 'in house', senior positions were not advertised, and the banks had a 'no-poaching' agreement with each other in relation to staff. Graduates have only been recruited in any numbers since the 1970 s. In Norway, banks traditionally recruited young people with little formal education, providing them with jobs and training within internal labour markets and industryspecific training via the Bank Academy. From the early 1960s, in both countries young women were recruited into banking as a secondary labour force, to carry out the routine tasks which were themselves increasingly subject to automation (Crompton 1989). The open gender exclusionary practices to be found in banking reflected the assumptions of the male breadwinner model, and in Britain, until well into the 1960 s men received a pay rise on marriage, and married women were transferred to separate grades. These practices were removed by equality legislation in the 1970s, but were nevertheless informally reproduced. For example, in Britain throughout the 7os and into the 8os young men - but not young women - were encouraged to take the Banks' professional examinations (essential for promotion).

These informal exclusionary practices were widely recalled by many of our interviewees, but there was also widespread agreement that on the whole, they were a thing of the past. Both national legislation, and the bank's own policies, have outlawed discrimination against women who aim to develop a career in banking. It 
is important not to be complacent that absolute gender equality has been achievedfor example, informal patterns such as 'old boys' networks can still be very important - but nevertheless, few would dispute that considerable progress has been made.

However, although bureaucratic careers may no longer be universally masculine, other pressures have been undermining the basis of the career itself. In both Britain and Norway, financial difficulties and crisis from the late 1980 s have resulted in massive restructurings and cutbacks of staff. What was once a 'job for life' has now become almost as insecure as other forms of employment. In Norway, the banking crisis resulted in a 22 per cent loss of jobs in the banking sector between 1987 and 1991, and job decline continues into the 9os. Seventy three per cent of those who lost their jobs at the time of the crisis were women, who often had only low levels of qualification. ${ }^{3}$ In Britain, the bank in which the interviews were conducted experienced a takeover in 1992, and a further round of restructuring in 1994. In some areas, staff were cut by as much as a third, and employees found themselves having to re-apply for their own jobs (similar policies have been implemented in Norwegian banks).

Growing insecurity of employment, therefore, has been a feature of work in banking in both Britain and Norway. In Britain, it was mainly older staff (over fifty) who were affected - indeed, some of those we interviewed suggested that they had survived restructuring because they were young and cheap. In Norway, however, protective legislation meant that the jobs of older staff were retained, and younger staff lost their jobs. Our Norwegian interviews, therefore, include criticisms and complaints as to the top-heavy age structure of the banking industry, and even with the small number of interviews conducted on the project, the Norwegian interviewees tended to be older than the British.

\section{Methodology, Data Collection, and Analysis}

'Gender' may be viewed as the set of relationships constructed around biological sex differences, and which serve to make these differences socially meaningful. Gender relations are not fixed, and vary according to circumstance and individual biography. Thus sex differences may be seen as the outcome of the processes through which gender relations are structured. The process of gender differentiation takes place within the totality of 'public' societal spheres (i.e. in the labour market, educational and welfare institutions, in organisations and public places - see Connell 1987), as well as at the family and interpersonal level. Although the presence of some kind of gender differentiation is universal, its characteristics vary from society to society, and in class, ethnic, and age groupings. Thus gender systems and their evolution need to be seen in their contexts if meaningful explanations and understandings are to be developed. A holistic, or case study, approach is therefore required in order to study gender processes. There is a 
longstanding contrast between 'case oriented' and variable oriented' approaches in the social sciences (Ragin and Zaret 1983, Ragin 1987, 1991), which has often been paralleled with 'qualitative' versus 'quantitative' methodologies. Whilst this parallel can be a valid one, it is also somewhat misleading in that whereas although it is true that a 'variable oriented' approach will always be quantitative, a case study may incorporate both quantitative and qualitative methods.

The logic of the comparative case study method has been extensively debated in historical sociology (see e.g. Skocpol and Somers 1980). It may be suggested that there are useful parallels to be drawn between historical case comparisons and the 'gender systems' approach to the study of gender relations (Crompton 1999a: 203ff). Gender systems approaches (and here we might mention the work of Connell 1987 Rubery et al. 1998, O’Reilly and Fagan 1998, Duncan 1995, Pfau-Effinger 1993 and Leira 1992 amongst others) recognise the complexity of the structuring of gender relations, as well as their outcomes. With the exception of Connell, all of the 'gender systems' authors mentioned above have grounded their arguments in comparative case study research.

There are a number of different logics of comparative case study research. Comparative research may be used to demonstrate the validity of a theoretical explanation via its repeated application across a range of different contexts - a strategy which is also associated with variable-oriented approaches (e.g. Kohn 1987). Other researchers have focused more on the difference between cases, or the contrast of contexts (Skocpol and Somers 1980), in order to understand divergent historical developments. Besides the work of gender systems researchers (see above) one might also mention here the debates deriving from Esping-Andersen's Three Worlds of Welfare Capitalism (1990). In this work, different types of welfare system are associated with different patterns of and consequences for social equality, women's employment, etc. Feminist scholars (e.g. Bock and Thane 1993, Lewis 1992, Sainsbury 1994) have criticised and developed Esping-Andersen's analysis, extending this contrast of contexts in order to incorporate the unpaid work of women, as well as the part played by the feminist movement in shaping welfare states.

Comparative case oriented research has also been used to develop causal explanations (Skocpol and Somers 1980, Ragin 1987, Pickvance 1995). These approaches have been developed out of J. S. Mill's logics of scientific explanation which include the 'method of agreement', the 'method of difference', and the 'indirect method of difference' (Mill 1961). There are a number of problems associated with Mill's methods, in particular the likelihood of multiple and plural causation which is itself linked to the impossibility of direct experimental manipulation in the social sciences and thus of establishing an uncontaminated 'case'. ${ }^{4}$ Nevertheless, in this paper we will use a variant of Mill's method of difference, as well as the contrast of contexts, in the analysis of biographical data collected in the two countries under discussion here. 
Biographical interviews with female bank managers in Britain (seventeen interviews) and Norway (fifteen interviews) were carried out during 1995-6 as part of the Gender Relations project. ${ }^{5}$ Interviews with male bank managers (sixteen in Britain and fifteen in Norway) were conducted in 1998-9. The aim of the project was to interview career staff, and therefore all interviewees were aged between thirty and fifty at the time of interview, and all were on managerial grades and employed in retail banking. A common interview guide was used to lead discussions which focused on family background, career decisions and entry into employment, employment careers and family life, etc. Interviews were taped, and relevant sections transcribed (and translated where necessary), then entered onto a common Recording Document.

The comparative strategy used in this paper is biographical matching. Each biography is treated as an individual 'case'. Four different matching exercises have been undertaken: Norwegian men and Norwegian women, British men and British women, Norwegian women and British women, and Norwegian men and British men. The main factor used in matching cases was age. Another important principle informing the matching process was the nature of the interviewees' qualifications and banking career. Individuals who had worked their way up 'though the ranks', having joined the bank at a young age with lower-level qualifications, were matched with similar individuals, and those with higher level qualifications were similarly matched. ${ }^{6}$ Finally, the nature of the interviewees' job has also been taken into account, and those in specialist or niche jobs - for example, IT - have been matched with interviewees in similar specialisms. ${ }^{7}$

This process of biographical matching, therefore, has compared similarities and differences within the matched pairs. As the matching exercise was carried out after the interviews had been completed (i.e. the study had not been designed with the intention of matching biographies exactly), not all cases could be matched with suitable equivalents. In any case, changes in both the bank's internal labour markets, as well as in attitudes to women, mean that there are some categories in which one sex will be over-represented even in a small number of interviews (e.g. men in midlife who have had a successful internal banking career). This difference was evident amongst the interviews carried out for the project, and further restricted matching possibilities. The final number of matches achieved was as follows: Norwegian women and British women, ten matches (Table 1), Norwegian men and British men, eight matches (Table 2), British men and British women, eleven matches, (Table 3 ) Norwegian men and Norwegian women, eleven matches (Table 4). For the sake of brevity, details of career paths are not given, it is simply reported whether they had been similar or different within the matches. More evidence is provided in the case of the particular examples discussed in the figures in the text.

The tables may be seen as a modified application of Mill's 'Method of difference'. The method of difference rests upon the comparison of cases which have different 
outcomes, but which resemble each other in all respects save a single factor which is then taken to be the cause of the outcome. Thus, for example, if individual cases in different countries are as near as possible identical in respects such as age, sex, educational level, circumstances of family of origin etc., but outcomes such as career structures or childcare patterns are different, then it is justifiable to suggest that national differences constitute the causal factor in these cases. Conversely, if outcomes do not vary (and as we shall see they do not), then national specifics are having less of an impact. A similar logic may be applied to male/female comparisons within the same country, but in this case, gender would be the explanatory (or 'independent') variable. As we shall see, the trends indicated in the tables are not absolutely consistent and it is not being argued that this serial application of the method of difference is an exact process. In order to develop further or support the arguments in this paper, exemplary biographical pairs have been abstracted and presented in more detail in the figures. These more detailed comparisons reveal both similarities and differences and in the case of the latter, may be seen as using the comparative logic of the 'contrast of contexts' in order to understand divergent outcomes.

\section{Gender Differences in Careers in Banking}

As noted above, in contrast to Britain, there is a recent history of woman and family friendly state policy making in Norway. Recent survey data suggests that gender role attitudes are more liberal in Norway than in Britain, and that Norwegian men are more likely to share domestic tasks than men in Britain (Crompton and Harris 1997). Nevertheless, in both Britain and Norway, the major contrast between the women and men interviewed was that the greater proportion of men, if they had had children, had lived with partners who had taken the responsibility for young children (twenty-five out of twenty-six men with children). In contrast, the women interviewed had fewer children, and most of them had taken the primary responsibility for care when the children were young (fifteen out of nineteen women with children) ${ }^{8}$

The first two columns of Table 1 summarise key biographical details of the matches between female bankers in Britain and Norway. The third column gives a summary indication of comparative organisational career paths, and the fourth column an indication of current domestic responsibility in each case (reading from left to right). (Similar conventions are followed in the other three tables.) It can be seen that despite the national differences noted above all of the female pairs had had similar careers - for example, all three matches born before 1950 had had 'late starting' careers. There was a predominance of sharing as far as current domestic responsibilities were concerned, reflecting the pressures that these managerial women were under (there had been a tendency for domestic sharing to increase as 


\section{Table 1}

\section{Female bankers: cross-national comparisons}

\begin{tabular}{|c|c|c|c|c|}
\hline & Norway & England & $\begin{array}{l}\text { Organisational } \\
\text { career }\end{array}$ & $\begin{array}{l}\text { Current } \\
\text { domestic } \\
\text { responsibility }\end{array}$ \\
\hline 1 & $\begin{array}{l}\text { 3/32: d.o.b. 1946, } \\
\text { children 1975, } 1977\end{array}$ & $\begin{array}{l}\text { 2/23: d.o.b. } 1946, \text { married, } \\
\text { no children }\end{array}$ & $\begin{array}{l}\text { similar late } \\
\text { starting careers }\end{array}$ & $\begin{array}{l}\text { negotiated } \\
\text { shared, } \\
\text { negotiated } \\
\text { shared }\end{array}$ \\
\hline 2 & $\begin{array}{l}\text { 3/29: d.o.b. 1948, } \\
\text { children 1969, } 1970\end{array}$ & $\begin{array}{l}\text { 2/33: d.o.b. } 1949 \text {, married, } \\
\text { no children, sick parents }\end{array}$ & $\begin{array}{l}\text { similar late } \\
\text { starting careers }\end{array}$ & $\begin{array}{l}\text { negotiated } \\
\text { shared, } \\
\text { negotiated } \\
\text { shared }\end{array}$ \\
\hline 3 & $\begin{array}{l}\text { 3/30: d.o.b. } 1948 \text {, } \\
\text { child 1983, } \\
\text { divorced, } 1988 .\end{array}$ & $\begin{array}{l}\text { 2/32: d.o.b. 1949, child } \\
\text { 1971, divorced } 1979\end{array}$ & $\begin{array}{l}\text { similar family } \\
\text { supporters, } \\
\text { relatively late } \\
\text { careers }\end{array}$ & self, self \\
\hline 4 & $\begin{array}{l}\text { 3/28: d.o.b. 1950, } \\
\text { children 1974, } 1977\end{array}$ & $\begin{array}{l}\text { 2/25: d.o.b. 1954, now } \\
\text { pregnant }\end{array}$ & similar & shared, shared \\
\hline 5 & $\begin{array}{l}\text { 3/31: d.o.b. } 1953 \\
\text { child } 1975\end{array}$ & $\begin{array}{l}\text { 2/24: d.o.b. } 1956 \text {, children } \\
1984,1988,1990\end{array}$ & $\begin{array}{l}\text { similar (niche } \\
\text { jobs) }\end{array}$ & $\begin{array}{l}\text { shared, } \\
\text { partner }\end{array}$ \\
\hline 6 & $\begin{array}{l}\text { 3/23: d.o.b. 1957, } \\
\text { children 1991, } 1994\end{array}$ & 2/37: d.o.b. 1961, child 1986 & similar & shared, partner \\
\hline 7 & $\begin{array}{l}\text { 3/21: d.o.b. 1959, } \\
\text { child adopted } 1993\end{array}$ & $\begin{array}{l}\text { 2/36: d.o.b. 1953, married, } \\
\text { MS diagnosis } 1993\end{array}$ & $\begin{array}{l}\text { similar: successful } \\
\text { but recent scaling } \\
\text { down }\end{array}$ & self, partner \\
\hline 8 & $\begin{array}{l}\text { 3/24: d.o.b. 1960, } \\
\text { unmarried }\end{array}$ & 2/30: d.o.b. 1959, child 1994 & $\begin{array}{l}\text { similar: successful } \\
\text { despite } \\
\text { discrimination }\end{array}$ & $\mathrm{n} / \mathrm{a}$, shared \\
\hline 9 & $\begin{array}{l}\text { 3/33: d.o.b. } 1960 \\
\text { children } 1985,1988 \\
\text { 1989, }\end{array}$ & $\begin{array}{l}\text { 2/26: d.o.b. } 1960 \text {, children } \\
\text { 1986, } 1988\end{array}$ & $\begin{array}{l}\text { similar careers, } \\
\text { different caring } \\
\text { arrangements }\end{array}$ & partner, self \\
\hline 10 & $\begin{array}{l}\text { 3/22: d.o.b. } 1965 \\
\text { no children }\end{array}$ & 2/28: d.o.b. 1963 , no children & similar (niche job) & $\mathrm{n} / \mathrm{a}, \mathrm{n} / \mathrm{a}$ \\
\hline
\end{tabular}

${ }^{1}$ Reading from left to right. Domestic responsibilities change over the family life cycle, and it is impossible to describe this variation in a single phrase. The tables, therefore, give an indication of current domestic responsibilities, these may have been different at other times. For example, some men have assumed responsibilities following separation from their partners, and women have renegotiated a more equal division of domestic tasks as their employment careers have become more time consuming. 
Table 2

Male bankers: cross national comparisons

\begin{tabular}{|c|c|c|c|c|}
\hline & Norway & England & $\begin{array}{l}\text { Organisational } \\
\text { career }\end{array}$ & $\begin{array}{l}\text { Current } \\
\text { domestic } \\
\text { responsibility }\end{array}$ \\
\hline 1 & $\begin{array}{l}\text { 3/81: d.o.b. 1947, } \\
\text { children 1974, } 1976\end{array}$ & $\begin{array}{l}\text { 2/74: d.o.b. 1951, children } \\
\text { 1988, } 1990\end{array}$ & similar & $\begin{array}{l}\text { partner, } \\
\text { partner }\end{array}$ \\
\hline 2 & $\begin{array}{l}\text { 3/80: d.o.b. } 1953 \text {, } \\
\text { children 1982, 1988, } \\
1990\end{array}$ & $\begin{array}{l}\text { 2/82: d.o.b. } 1956 \text {, children } \\
\text { 1986, } 1990\end{array}$ & $\begin{array}{l}\text { similar successful } \\
\text { careerists }\end{array}$ & $\begin{array}{l}\text { partner, } \\
\text { partner }\end{array}$ \\
\hline 3 & $\begin{array}{l}\text { 3/76: d.o.b.1954, } \\
\text { children 1978, 1980, } \\
\text { split 1987. }\end{array}$ & $\begin{array}{l}\text { 2/84: d.o.b. 1952, children } \\
\text { 1979, 1980, split } 1990\end{array}$ & $\begin{array}{l}\text { similar, lateish start, } \\
\text { different care } \\
\text { patterns }\end{array}$ & self, self \\
\hline 4 & $\begin{array}{l}\text { 3/74: d.o.b. } 1958, \\
\text { children 1986, } 1988\end{array}$ & $\begin{array}{l}\text { 2/75: d.o.b. 1956, children } \\
\text { 1984, } 1986\end{array}$ & $\begin{array}{l}\text { similar stalled } \\
\text { careers }\end{array}$ & $\begin{array}{l}\text { partner, } \\
\text { partner }\end{array}$ \\
\hline 5 & $\begin{array}{l}\text { 3/75: d.o.b. 1959, } \\
\text { children 1986, 1988, } \\
1992\end{array}$ & $\begin{array}{l}\text { 2/73: d.o.b. 1957, children } \\
\text { 1984, 1986, } 1990\end{array}$ & similar & $\begin{array}{l}\text { partner, } \\
\text { partner }\end{array}$ \\
\hline 6 & $\begin{array}{l}\text { 3/79: d.o.b. 1961, } \\
\text { child } 1996\end{array}$ & $\begin{array}{l}\text { 2/72: d.o.b. } 1958, \text { married } \\
\text { no children }\end{array}$ & similar & partner, n/a \\
\hline 7 & $\begin{array}{l}\text { 3/73 d.o.b. 1962, } \\
\text { children 1992, } 1995\end{array}$ & $\begin{array}{l}\text { 2/76: d.o.b. } 1963 \text {, children } \\
\text { 1994, } 1997\end{array}$ & $\begin{array}{l}\text { similar, } \\
\text { different care } \\
\text { patterns }\end{array}$ & shared, shared \\
\hline 8 & $\begin{array}{l}\text { 3/77: d.o.b. } 1963 \text {, } \\
\text { children 1986, 1990, } \\
\text { 1992, divorced }\end{array}$ & $\begin{array}{l}\text { 2/79: d.o.b. } 1961 \text {, divorced } \\
\text { no children }\end{array}$ & similar & shared, n/a \\
\hline
\end{tabular}

${ }^{1}$ See footnote Table 1

female careers developed), although, as we have seen in the previous paragraph, most women had taken the main responsibility when children were very young.

The careers of the male bankers in the two countries had also been very similar, as can be seen from Table 2 (where careers are simply described as 'similar', this indicates that the pair in question had had similar, orthodox, linear, careers). In some contrast to the female bankers, in most cases the (female) partners of male bankers still took responsibility for domestic tasks. However, as we shall see in our later discussion, there were some interesting deviants from this pattern. 


\begin{tabular}{|c|c|c|c|c|}
\hline & Women & Men & $\begin{array}{l}\text { Organisational } \\
\text { career }\end{array}$ & $\begin{array}{l}\text { Current } \\
\text { domestic } \\
\text { responsibility }^{1}\end{array}$ \\
\hline 1 & $\begin{array}{l}\text { 2/36: d.o.b. } 1953 \text {, } \\
\text { married, MS } \\
\text { diagnosis } 1993\end{array}$ & $\begin{array}{l}\text { 2/82: d.o.b. 1956, } \\
\text { children 1986, } 1990\end{array}$ & similar & partner, partner \\
\hline 2 & $\begin{array}{l}\text { 2/25: d.o.b. 1954, } \\
\text { now pregnant }\end{array}$ & $\begin{array}{l}\text { 2/74: d.o.b. } 1951 \text {, } \\
\text { children } 1988,1990\end{array}$ & similar & shared, partner \\
\hline 3 & $\begin{array}{l}\text { 2/35: d.o.b. 1955, } \\
\text { child }\end{array}$ & $\begin{array}{l}\text { 2/75: d.o.b. 1956, } \\
\text { children 1984, } 1986\end{array}$ & different & self, partner \\
\hline 4 & $\begin{array}{l}\text { 2/30: d.o.b. 1959, } \\
\text { child } 1994\end{array}$ & $\begin{array}{l}\text { 2/73: d.o.b. 1957, } \\
\text { children 1984, 1986, } 1990\end{array}$ & different & shared, partner \\
\hline 5 & $\begin{array}{l}\text { 2/26: d.o.b.1960, } \\
\text { children 1986, } 1988\end{array}$ & $\begin{array}{l}\text { 2/86: d.o.b. } 1963 \text {, children } \\
\text { 1991, 1993, } 1996\end{array}$ & different & self, partner \\
\hline 6 & $\begin{array}{l}\text { 2/37: d.o.b. } 1961 \text {, } \\
\text { child } 1986\end{array}$ & $\begin{array}{l}\text { 2/78: d.o.b. } 1960, \text { married } \\
\text { no children }\end{array}$ & similar & partner, partner \\
\hline 7 & $\begin{array}{l}\text { 2/28: d.o.b. } 1963 \text {, } \\
\text { no children }\end{array}$ & $\begin{array}{l}\text { 2/85: d.o.b. 1967, children } \\
\text { 1991, } 1996\end{array}$ & similar & n/a, partner \\
\hline 8 & $\begin{array}{l}\text { 2/34: d.o.b. } 1963 \\
\text { no children }\end{array}$ & $\begin{array}{l}\text { 2/77: d.o.b. 1961, } \\
\text { no children }\end{array}$ & similar & $\begin{array}{l}\mathrm{n} / \mathrm{a}, \mathrm{n} / \mathrm{a}(\mathrm{both} \\
\text { shared) }\end{array}$ \\
\hline 9 & $\begin{array}{l}\text { 2/21: d.o.b. 1964, } \\
\text { child } 1993\end{array}$ & $\begin{array}{l}\text { 2/71: d.o.b. } 1967 \text {, } \\
\text { child } 1996\end{array}$ & different & shared, partner \\
\hline 10 & $\begin{array}{l}\text { 2/22: d.o.b. } 1965 \text {, } \\
\text { no children }\end{array}$ & $\begin{array}{l}\text { 2/83: d.o.b. 1964, } \\
\text { not married/children }\end{array}$ & similar & $\mathrm{n} / \mathrm{a}, \mathrm{n} / \mathrm{a}$ \\
\hline
\end{tabular}

${ }^{1}$ See footnote Table 1

The by-sex comparisons between countries, therefore, reveal similarities between women, and men, irrespective of national origin. This suggests that gender is the most significant causal factor in determining both organisational careers and domestic responsibilities. This conclusion (which is not surprising) is reinforced by the within-country gender comparisons in Tables 3 and 4. In these tables, 'similar' in the third column indicates that the matches both had orthodox linear careers, 'different' indicates that the woman's career had either started late or suffered an 
Table 4

Norwegian bankers: gender comparisons

\begin{tabular}{|c|c|c|c|c|}
\hline & Women & Men & $\begin{array}{l}\text { Organisational } \\
\text { career }\end{array}$ & $\begin{array}{l}\text { Domestic } \\
\text { responsibility }\end{array}$ \\
\hline 1 & $\begin{array}{l}\text { 3/27: d.o.b. } 1943 \\
\text { children 1962, 1965, } \\
\text { 1967, separated since } \\
1985\end{array}$ & $\begin{array}{l}\text { 3/82 d.o.b. 1946, } \\
\text { children 1972, 1974, } 1984\end{array}$ & different & self, partner \\
\hline 2 & $\begin{array}{l}\text { 3/29: d.o.b. } 1948, \\
\text { children } 1969,1970\end{array}$ & $\begin{array}{l}\text { 3/87: d.o.b. 1947, } \\
\text { children }\end{array}$ & different & self, shared \\
\hline 3 & $\begin{array}{l}\text { 3/32: d.o.b. 1946, } \\
\text { children 1975,1977 }\end{array}$ & $\begin{array}{l}\text { 3/81: d.o.b. 1947, } \\
\text { children 1974, } 1976\end{array}$ & different & shared, partner \\
\hline 4 & $\begin{array}{l}\text { 3/30: d.o.b. 1948, } \\
\text { child 1983, divorced }\end{array}$ & $\begin{array}{l}\text { 3/84: d.o.b. } 1953 \text {, } \\
\text { child 1979, divorced }\end{array}$ & similar & self, partner \\
\hline 5 & $\begin{array}{l}\text { 3/28: d.o.b. } 1950, \\
\text { children 1974, } 1977\end{array}$ & $\begin{array}{l}\text { 3/76 d.o.b. 1954, children } \\
\text { 1978, 1980, split } 1987\end{array}$ & $\begin{array}{l}\text { similar (but both } \\
\text { irregular) }\end{array}$ & shared, self \\
\hline 6 & $\begin{array}{l}\text { 3/25 d.o.b. 1953: } \\
\text { children 1984, 1986, } \\
1990\end{array}$ & $\begin{array}{l}\text { 3/74: d.o.b. } 1958, \\
\text { children 1986, } 1988\end{array}$ & different & self, partner \\
\hline 7 & $\begin{array}{l}\text { 3/21: d.o.b. } 1959 \text {, } \\
\text { child adopted } 1993 .\end{array}$ & $\begin{array}{l}\text { 3/75: d.o.b. 1959, } \\
\text { children 1986, 1988, } 1992\end{array}$ & similar & self, partner \\
\hline 8 & $\begin{array}{l}\text { 3/23: d.o.b. 1957, } \\
\text { children 1991, } 1994\end{array}$ & $\begin{array}{l}\text { 3/71: d.o.b. 1958, children } \\
\text { 1986, 1989, 1990, } 1993\end{array}$ & similar & shared, shared \\
\hline 9 & $\begin{array}{l}\text { 3/26: d.o.b. } 1959 \text {, } \\
\text { children 1989, } 1993\end{array}$ & $\begin{array}{l}\text { 3/75: d.o.b. } 1959, \\
\text { children 1986, 1988, } 1992\end{array}$ & similar & shared, partner \\
\hline 10 & $\begin{array}{l}\text { 3/24: d.o.b. 1960, } \\
\text { unmarried, no } \\
\text { children }\end{array}$ & $\begin{array}{l}\text { 3/73 d.o.b. 1962, children } \\
\text { 1992,1995 }\end{array}$ & similar & n/a, shared \\
\hline 11 & $\begin{array}{l}\text { 3/33: d.o.b. } 1960 \text {, } \\
\text { children } 1985,1988, \\
1989\end{array}$ & $\begin{array}{l}\text { 3/78: d.o.b. 1965, children } \\
\text { 1994, } 1996\end{array}$ & similar & partner, partner \\
\hline
\end{tabular}

${ }^{1}$ See footnote Table 1

interruption (e.g. extended maternity leave, child-rearing breaks, and/or part-time working). Table 3 indicates that six of the British women bankers had had similar organisational careers to their male comparators, and four were different. 
Interestingly, when men and women had experienced similar career paths, then their domestic responsibilities tended to be similar as well - that is, domestic responsibilities had been shared, or taken on by the partner, and/or the women in question were childless. A similar pattern emerges from the Norwegian gender comparisons (Table 4). ${ }^{9}$ These patterns illustrate the essential interdependence between 'employment' and 'domestic' career paths.

Many of the biographical matches, therefore, told a similar story, and the past history of both domestic and banking careers in both countries is illustrated in the following three examples. Figure 1 compares a Norwegian man and woman of similar age, both of whom had worked their way up through the hierarchy of the bank. However, whereas the female respondent has achieved this despite a career break, the man's wife was the primary carer of their children. Similarly, Figure 2 describes two younger bank employees who have both been careerists, but whereas the woman has modified her career ambitions with the arrival of her child, the man is explicit that his family has had no impact on his career trajectory.

The third example, from Britain, describes a matching of younger cases. As with the matches described in Figures 1 and 2, the man's wife has assumed the major responsibility for childcare, but childcare is shared in the case of the female interviewee. This suggests that over time, men's contribution to caring work is on the increase - at least among women who are in full-time employment.

\section{National Differences}

The contrasts between male and female careers in banking and the family, therefore, would appear to follow a similar pattern in both Britain and Norway.

Figure 1

\section{Older managers in Norway: gender comparisons}

\begin{tabular}{|l|l|}
\hline \multicolumn{2}{|c|}{ Norway } \\
\hline Woman & Man \\
\hline 3/27: group leader ${ }^{10}$ & 3/82: area leader \\
d.o.b. 1943. children 1962, 1965, 1967, separated & d.o.b. 1946. Married 1971, children 1972, 1974, \\
since 1985. Working-class family. Entered bank & 1984. Working-class background but \\
because it was a 'good' job. Home for 9 years & encouraged at school, worked his way up \\
with children, then part-time for 3 years, while & taking bank exams. Wife at home (8 years) \\
her sister cared for youngest child. Took Bank & when the children were young, he travelled a \\
Academy by correspondence course, working & lot, applied for new posts every two years or so \\
after children slept, with no support from & to get experience. Now his wife works full-time \\
husband. Pro-active applying for positions of & and travels a lot herself. He is looking towards \\
responsibility. Now looking towards & retirement. \\
retirement. & \\
\hline
\end{tabular}


Figure 2

Younger managers in Norway: gender comparison

\begin{tabular}{|l|l|}
\hline \multicolumn{2}{|c|}{ Norway } \\
\hline Woman & Man \\
\hline 3/21: assistant manager & 3/75: assistant director \\
d.o.b. 1959. Married 1978, child adopted 1993. & d.o.b. 1959. Married 1983, children 1986, 1988, \\
Got business degree, became salesperson for & 1992. Economics from college, training in \\
computer company, then shifted to bank in & accountancy. Joined the bank in his mid 20. \\
mid 2os. Ambitious prior to child, now & Career:'I have used the possibilities which \\
tempered: 'My own ambitions have fallen since & arose - never said “no'.' Meritocrat'... you \\
becoming a mother, I don't want too much & have to take the best people, male or female'. \\
responsibility'. No longer puts so much into & Wife (nurse) works part-time, children also \\
her job, feels guilt, but flexible so can take work & went to kindergarten. 'Family life has not \\
home. Expected equal sharing of housework & restricted my work career ... the last two years \\
and childcare, but has not got it. & I feel as though I have had two jobs but if one is \\
& to go upwards then it demands something.' \\
\hline
\end{tabular}

However, in respect of their national approaches to the gender question, and childcare policies, the recent history of the two countries has been rather different. In the 1980s, it was suggested that a 'woman friendly' Norwegian state might redress the imbalances of previous patriarchal decades (Hernes 1987), and the state has also introduced a number of 'family friendly' legislative measures that have been well documented (Ellingsaeter and Hedlund 1998, Brandth and Kvande 1999). Parents are entitled to up to thirty-nine weeks of paid leave, of which four weeks are set aside for the father (leave is forfeited if not taken by the father). This is in addition to statutory maternity

Figure 3

\section{British managers in their thirties - gender comparison}

\begin{tabular}{|l|l|}
\hline \multicolumn{2}{|c|}{ Britain } \\
\hline Woman & Man \\
\hline 2/21: D4 & 2/71:D4 \\
d.o.b. 1964. First marriage 1982-90, second & d.o.b. 1967. Married 1993, child 1996. Father \\
marriage 1992, child 1993. Father driving & driver, mother part-time secretary. Joined \\
instructor, mother secretary. 11 O levels- & bank after O levels because of rising \\
refused to stay on for A levels because she & unemployment - it was a secure job. \\
wanted to leave school and home. Got a job in a & Encouraged to take bank qualification, got \\
bank, married at 18. When she started (1980) & transferred to MDP (Management \\
there was no encouragement for girls to take & Development Programme). His wife also \\
exams, but she insisted. Did 2 year BTEC & worked for the bank, she lost out in \\
national, then banking exams - a long haul & restructuring so she took a redundancy \\
which she only finished in 1989. Grew apart & package to stay at home with their daughter. \\
from her husband. Remarried to another bank & Wife now full-time carer. \\
manager. Domestic work and childcare shared. & \\
\hline
\end{tabular}


leave, entitlements to leave to care for sick children, short-term 'daddy leave' and unpaid entitlements that can extend up to two years. Childcare provision is also more extensive than in Britain. The national context, therefore, is much more favourable in Norway as far as the combination of employment and caring is concerned. However, although national circumstances might not be particularly advantageous in the British case, the bank in which the British interviews were carried out has a high profile as a 'family-friendly' employer, having taken the lead with innovations such as workplace nurseries, leave to care for sick children, and so on.

The impact of these national differences has been explored though an examination of the eighteen cross-national matches in Tables 1 and 2. As has been discussed in the previous section, most of the matches revealed rather similar work-family patterns as between men and women in the two countries, suggesting that national differences are not particularly important in this regard. Indeed, the biographies reveal many parallels between the two countries. Figure 4 illustrates the negative impact of bank restructuring on the career paths of some older men, and in both cases, it may be suggested that the men in question have coped with career disappointment by retreating into family life. In contrast, Figure 5 suggests that men who have continued in successful careers despite restructuring have done so at some personal and family cost.

The women's matched biographies also demonstrated significant continuities. Figure 6 describes the careers of two women who both began their bank careers in the 1970 - that is, after the passing of equal opportunities and anti-discrimination legislation. Nevertheless, it illustrates the discrimination that women in banking faced even in these relatively recent times.

\section{Figure 4}

\section{The impact of bank restructuring: stalled careers}

\begin{tabular}{|l|l|}
\hline \multicolumn{2}{|c|}{ Men } \\
\hline Norway & Britain \\
\hline 3/74: was divisional leader & 2/75: D4 \\
d.o.b.1958. Married 1980, children 1986, 1988. & d.o.b. 1956. Married 1979, children 1984, 1986. \\
$\begin{array}{l}\text { Commercial school. Has worked up from } \\
\text { cashier, Banking Academy Diploma. Wife was }\end{array}$ & Grammar school, A level, straight into bank \\
a secretary, at home when the children were & Banking Exams. Wife cared for children (self- \\
young, now works as an assistant at their & library. He has lost out in reorganisation, he \\
children's school. Career prospects affected by & was in foreign investments which he enjoyed \\
bank restructuring: 'No one is permanent in & but the section was moved. His life is outside \\
the bank any more.' He was a divisional leader & the bank-caravanning, football supporter, \\
for region, now'works on projects'.'The & swimming rallies with his children (he is an \\
children have activities every day, the boy plays & instructor). \\
football and I am a trainer'. & \\
\hline
\end{tabular}


Figure 5

The impact of bank restructuring: successful careers

\begin{tabular}{|c|c|}
\hline \multicolumn{2}{|c|}{ Men } \\
\hline Norway & Britain \\
\hline $\begin{array}{l}\text { 3/75: assistant director } \\
\text { d.o.b. 1959. Married 1983, children 1986, 1988, } \\
\text { 1992. Economics from college, training in } \\
\text { accountancy. Joined the bank in his mid } 20 s . \\
\text { Career:'I have used the possibilities which } \\
\text { arose-never said "no'”. Meritocrat ... you } \\
\text { have to take the best people, male or female'. } \\
\text { Wife (nurse) works part-time, children also } \\
\text { went to kindergarten.'Family life has not } \\
\text { restricted my work career ... the last two years } \\
\text { I feel as though I have had two jobs but if one is } \\
\text { to go upwards then it demands something. }\end{array}$ & $\begin{array}{l}\text { 2/73: D5 } \\
\text { d.o.b. 1957. Married 1981, children 1984, 1986, } \\
\text { 1990. Took A levels at college. Did well in the } \\
\text { bank exams, got first Appointment at age } 25 \\
\text { (young), sent to a very difficult job that he did } \\
\text { well: '... I did three separate jobs within } 18 \\
\text { months and that taught me a lot about politics } \\
\text { at board level.' He has survived restructuring. } \\
\text { Wife responsible for childcare and house, she } \\
\text { does occasional part-time work. His job means } \\
\text { he has to work irregular hours and entertain: } \\
\text { 'The sort of job I'm in, if a customer says jump } \\
\text { you jump.' }\end{array}$ \\
\hline
\end{tabular}

Our biographical comparisons, therefore, suggest that the employment and family careers of bank workers in Britain and Norway are very similar, despite the difference in gender and family policies in the two countries. However, there were two contrasting cases, both male matches, that did suggest that different national policies can have a very important impact in particular instances. Figure 7 illustrates the experiences of two men who have both, albeit for rather different reasons, taken

Figure 6

Women in bank management: national comparisons

\begin{tabular}{|c|c|}
\hline \multicolumn{2}{|c|}{ Women } \\
\hline Norway & Britain \\
\hline $\begin{array}{l}\text { 3/24: financial consultant } \\
\text { d.o.b. } 1960 . \text { In a relationship, no children. } \\
\text { Mother housewife, father engineer. Private } \\
\text { school, sister bright and role model. Degree in } \\
\text { finance. Held a number of positions in bank: 'I } \\
\text { got to be a branch manager but there is a lot of } \\
\text { overtime and meetings ... I had to give a lot of } \\
\text { myself.' Wants promotion, has good } \\
\text { qualifications and possibilities. Has faced } \\
\text { discrimination: 'The "good old boys" } \\
\text { absolutely exists. Women are a threat to their } \\
\text { integrity. Women have to be better than men to } \\
\text { achieve as much. Men are unwilling to let } \\
\text { women into their territory'. }\end{array}$ & $\begin{array}{l}\text { 2/30: D5 } \\
\text { d.o.b. 1959. Married 1981, child 1994. Father } \\
\text { teacher, mother housewife, both encouraged } \\
\text { achievements. Academically successful. } \\
\text { Entered banking with father's encouragement } \\
\text { at } 18 \text {. Completed ACIB by } 21 \text { (very young). } \\
\text { Throughout career has met with } \\
\text { discrimination: 'I went to one of my line } \\
\text { managers and said I wanted to do foreign ... he } \\
\text { said 'Clare, you're a woman, you will never go } \\
\text { on foreign ... I was so bloody angry about it } \\
\text { that I thought 'right, you swines', and to me, } \\
\text { the way to show them was to keep succeeding.' } \\
\text { Feels she's lost } 3 \text { years of career through having } \\
\text { a child. }\end{array}$ \\
\hline
\end{tabular}


Figure 7

Men with caring responsibilities: national comparisons

\begin{tabular}{|c|c|}
\hline \multicolumn{2}{|c|}{ Men } \\
\hline Norway & England \\
\hline $\begin{array}{l}\text { 3/73: assistant director } \\
\text { d.o.b. 1962. Married 1988, children 1992, } 1995 . \\
\text { Wife a teacher, she works } 75 \text { per cent of time. } \\
\text { Has slowed down career because of his family. } \\
\text { 'The first time I had paternity leave I had three } \\
\text { months, the next time I had four and a half } \\
\text { months ... I do not feel that I have enough } \\
\text { time with the children and I have been on } \\
\text { shorter working hours a couple of times - } \\
\text { varied between } 80 \text { per cent and } 90 \text { per cent ... } \\
\text { Not all managers are positive to reduced } \\
\text { working hours - but the bank is big and they } \\
\text { often know well in advance when leave or } \\
\text { reduced hours is required and it is easy to } \\
\text { arrange for deputy staff. The possibility is } \\
\text { there if one has the will.' }\end{array}$ & $\begin{array}{l}\text { 2/76:D4 } \\
\text { d.o.b. } 1963 . \text { Married 1983, children 1994, } 1997 . \\
\text { Difficult family circumstances - sick child, } \\
\text { wife had breakdown, he finds himself having } \\
\text { to do shopping and housework at weekends } \\
\text { despite working long hours. 'Employers expect } \\
\text { a lot of you - you can't sit there from } 9 \text { to } 5 \\
\text { without any pressure. So they've been put } \\
\text { under more pressure ... I think it's put the } \\
\text { family unit under so much stress ... With them } \\
\text { watching the profit line constantly they're not } \\
\text { willing to put extra manpower, planning and } \\
\text { resourcing into the network to relieve those } \\
\text { strains.' }\end{array}$ \\
\hline
\end{tabular}

on an enhanced level of family responsibilities. The Norwegian interviewee has found it possible to take extended leave, and to reduce his working hours, whereas the British interviewee (whose case is discussed in further detail below), has not found it possible to do this - despite the ostensibly 'family-friendly' policies of the bank in question.

An even more striking national contrast is illustrated in Figure 8. In both cases, the men had become the sole carers of their children as a consequence of a marriage break-up. However, whereas the Norwegian interviewee seems to have been (almost) overwhelmed with public assistance, the British interviewee received none at all.

To summarise our findings so far: a comparison of the domestic and organisational careers of men and women in banking in Norway and Britain suggests that, despite the very real national differences in attitudes and policies between the two countries, the work-family experiences of the men and women are very similar, and moreover, are in some contrast with each other. Nevertheless, the contrasting experiences of men for whom childcare responsibilities were significant does suggest that a pro-active national policy can 'make a difference'. However, this would seem to be a difference at the margins, and it is of interest that it has been experienced by somewhat atypical men, rather than women. It is important to emphasise that this should not be taken as an argument to the effect that as government policies in this area appear to have a relatively minor impact, then there is no point in arguing for state policies that seek to change the balance of domestic labour between men and 
Figure 8

Male sole carers: national comparisons

\begin{tabular}{|c|c|}
\hline \multicolumn{2}{|c|}{ Men } \\
\hline Norway & Britain \\
\hline $\begin{array}{l}\text { 3/76: financial consultant } \\
\text { d.o.b. 1954. Married 1977, children 1978, 1980, } \\
\text { split 1987. Childcare before split: wife partly at } \\
\text { home, kindergarten.'I had an } 80 \text { per cent job } \\
\text { because of the kids - one of just a few men with } \\
\text { this advantage. I moved to another part of } \\
\text { town to get a free kindergarten place ... the } \\
\text { youngest got an infectious disease and had to } \\
\text { be home for } 3-8 \text { weeks. I had help from the } \\
\text { social office ... after that I had a commune } \\
\text { home help once a week. I don't think a woman } \\
\text { would have got that.' Not interested in career. }\end{array}$ & $\begin{array}{l}\text { 2/84: } \mathrm{D} 4 \\
\text { d.o.b.1952. Married 1972, children 1979, 1980, } \\
\text { split } 1990 \text {. Wife responsible for childcare } \\
\text { before split. Interviewee became sole carer, no } \\
\text { public or private assistance: 'All my family was } \\
\text { in Scotland, her family was in Newcastle so } \\
\text { basically there was nobody to help ... to be } \\
\text { honest in the early days it was just the three of } \\
\text { us ... they were latchkey kids.' His career has } \\
\text { been adversely affected as he is unable to be } \\
\text { mobile. }\end{array}$ \\
\hline
\end{tabular}

women, or between employment and family life. As the exceptional cases demonstrate, family friendly legislation and child-care provision can make a considerable difference. Nevertheless, there remains the wider question of the actual extent to which the state can really counteract the family unfriendly demands of market forces in a competitive business environment.

\section{'At the end of the day the profit margin is most important'}

The 'male breadwinner' model separated responsibilities for caring and social reproduction from the demands of the market, and indeed, the development of the model was accompanied by the growth of an ideology of the absolute separation of the 'public' (market) and 'private' (domestic) spheres, and the different sets of values associated with them - despite the fact of their mutual interdependence. Although it is widely acknowledged that the growth of married women's employment represents a practical shift from a single (male) to a dual earner model of employment and family life, it would seem that it is taking some time for a corresponding shift in values and behaviour to be achieved.

In this paper we have focused on career jobs in the private sector. Ellingsaeter's discussion of the Norwegian case (1999) has highlighted the importance of sectoral location as far as moves towards a dual earner/dual carer model of employment and family life is concerned. Not only do public sector workers in Norway have better parental rights, but fathers are more likely to take parental leave, and flexible working is more widespread. The greater market orientation of the private sector, therefore, is likely to work against the implementation of 'family friendly' policies, whatever the 
rhetoric of governments and employers might be. As one of our interviewees (who, it will be remembered, was employed by a 'family friendly' work organisation) put it:

... although my manager said to me 'take as much time as you like' [when his daughter was born with health problems], I was given the message ... how busy the office was - and I was expected to be back at work ... at the end of the day the profit margin is the most important ... Unless you get your life sorted out you're not going anywhere, you'll just end up being downgraded and possibly they'll want to get rid of you $(2 / 76$, see Figure 7 above).

Indeed, there were no examples in any of our interviews, amongst either women or men, of dual earner partnerships where both people had maximised their career objectives. The conflicts between the demands of a successful career and family life are well illustrated in the two examples that follow. Figure 9 describes the biographies of two successful Norwegian careerists whose partners have taken the major responsibility for childcare, to the detriment of their own careers.

Figure 10 illustrates a parallel British case (although it should be noted that in this example the interviewees had worked their way up from a relatively low qualification base). A comparison of Figures 9 and 10 does suggest that the pressures in the British bank were even greater than in the Norwegian bank - and indeed, the last round of restructuring had been more recent in the British bank. Nevertheless, this point does not significantly undermine our more general arguments as to the inherent difficulties of combining employment with family life, even in the most favourable of national circumstances.

Figure 9

\section{Succesful careers in Norway: gender comparison}

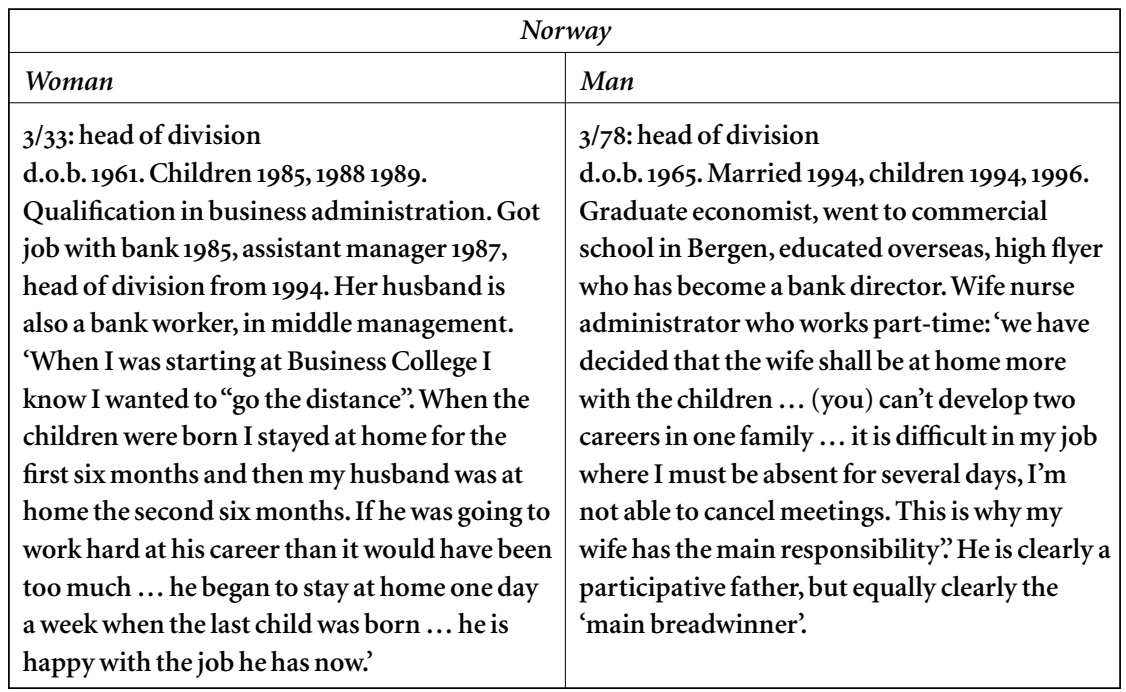


Since the Second World War, the changes in the employment circumstances of women have been extremely rapid - compare, for example, the biographies of the women in Figures 1 and 3 (although even in the latter case, the interviewee initially faced organisational discouragement). These changes have been officially recognised at both the national and international levels. For example: 'More women - and also more men - are facing dual and often conflicting labour market and family responsibilities. Ensuring the compatibility of employment and family commitments within individual lives is a major challenge emerging from the process of structural change' (OECD 1994:1). However, an in-depth examination of a number of these 'individual lives' suggests that this challenge is often not being met, even in relatively favourable circumstances.

The reconciliation of employment and family life is not a problem for which there is likely to be a single solution in practice. Just as the 'male breadwinner' model was never a universal phenomenon, either at the level of the nation-state or in individual families (Pfau-Effinger 1993), so a variety of national and personal

Figure 10

Employment - family conflict in Britain: gender comparison

\begin{tabular}{|c|c|}
\hline \multicolumn{2}{|c|}{ Britain } \\
\hline Woman & Man \\
\hline $\begin{array}{l}\text { 2/37: senior D4 } \\
\text { d.o.b. 1961, Married 1983, child 1986. Working- } \\
\text { class family. Bright at school but left at O level. } \\
\text { Fell into bank at sixteen for money. Began } \\
\text { working way up through clerical grades, had } \\
\text { baby, and returned to find she'd lost } \\
\text { promotion because of maternity leave - that } \\
\text { 'kick started' her career: 'It took me to twenty- } \\
\text { five to really wake up.' Restructuring of } \\
\text { banking and shift to more sales orientated } \\
\text { approach provided her with opportunities to } \\
\text { work her way up. First managerial } \\
\text { appointment in } 1989 \text {.'I used to see customers } \\
\text { from dawn to dusk, and I performed, I was } \\
\text { very successful, but I was tired and the family } \\
\text { went apart...' Returned to branch } \\
\text { management where more regular schedules. } \\
\text { Intended to have second child but has been } \\
\text { unable to fit it in with work. She is the main } \\
\text { breadwinner. Husband was made redundant, } \\
\text { now he works part-time three nights a week, to } \\
\text { be with their daughter after school. }\end{array}$ & $\begin{array}{l}\text { 2/78: D5 } \\
\text { d.o.b.1960. Married 1983, no children. } \\
\text { Working-class family, grammar school O } \\
\text { levels. Got a job in banking, did banking } \\
\text { exams, rose through the ranks. Works very } \\
\text { hard-one of reasons wife went part-time is } \\
\text { that he can't help with housework: 'We were } \\
\text { both finding ourselves absolutely totally worn } \\
\text { out working full-time. I'm still worn out } \\
\text { working full time but at least she's relaxed } \\
\text { when I get home so we can have some quality } \\
\text { time together ... Management grades don't get } \\
\text { paid overtime, we're being asked to do such a } \\
\text { lot more, take on so much more responsibility } \\
\ldots \text { I wouldn't think the bank as an organisation } \\
\text { really shows much concern for an individual's } \\
\text { private life. Their concern is getting the work } \\
\text { done to maximise the income at the least cost.' }\end{array}$ \\
\hline
\end{tabular}


strategies may and will be developed in adapting to a dual breadwinner situation. Nevertheless, it is important to recognise that different resolutions of employment and caring will tend to have different outcomes for both gender relations as well as wider systems of inequality (see Crompton 1999a, chapter 10). The job discussed in this paper - bank management - is almost invariably a full-time occupation. Parttime work for women is a major strategy through which dual earning and families can be managed, but also tends to be associated with the persistence of male dominance in gender relations in the household and paid employment (part-time work for both partners would be another matter). ${ }^{11}$ Marketised caring - as has been developed in the UK and the US - has been associated with widening inequalities in society as a whole. The provision of substitute care by the state, particularly, as in the Scandinavian countries, in a context informed by second-wave feminism, has been associated at the aggregate level with the narrowing of gender inequalities and no substantial increase in societal inequality. Nevertheless, our findings tend to confirm Ellingsaeter's suggestions as to the limitations of 'state feminism' when confronted by market forces.

For the logic of the explanation of the failure to meet the challenge of ensuring the compatibility of employment and family commitments in many of the individual lives we have studied is embarrassingly simple. Pressures towards economic competitiveness have generated organisational restructuring (our discussion has focused on the financial sector but there are in fact few areas of employment where this is not the case), these pressures have been passed on to individuals who, if they wish to improve their chances of a successful career, are constrained to behave in ways that can make family life difficult. As Figures 9 and 10 demonstrate, these pressures may be eased if a partner is available to absorb them - and the partner may be male or female. ${ }^{12}$ Individuals - men and women - may choose to put less into their careers, but this choice is likely to stall their career development. Other individuals may find their careers stalled for them, as in the examples in Figure 4. Thus a model of employment is perpetrated in which the 'best' jobs also happen to be those least compatible with employment and caring. For as long as the model of a competitive market is taken as a template for organisational structuring, then this process will be repeated. Indeed, recent innovations in managerial strategy, such as Total Quality Management (which promotes internal competition within the organisation), together with the increase in individualised and performance related pay, etc., do not augur well for the growth of family friendly employment. ${ }^{13}$

In this paper, we have not sought to suggest that the family friendly policies pursued in Norway and other Scandinavian social democracies have not been important, nor that they have not had a positive effect. They have done, and the Scandinavian example continues, rightly, to be cited as 'best practice' by organisations lobbying for change in Britain and elsewhere (e.g. New ways to Work 1995). The exceptional cases of male carers identified in our research might indeed confirm, as 
Brandth and Kvande have argued, the success of directive state feminism in assisting and perhaps changing (some) men in Norway. Nevertheless, our aim has been to point to the de facto incompatibility between family life and full-time careers in the competitive market sector of employment, an incompatibility which seems to have been little affected by recent shifts in government and employer policies. This suggests that it is necessary to maintain a continuing awareness of the tension between market forces and social reproduction. The need for a shift in the balance of emphasis as between the two would seem to be obvious, but this need as yet continues to be outweighed by economic and other organisational priorities.

NOTES

1. As Leira (1992) has demonstrated, the provision of care for younger children in Norway lagged behind that of the other Scandinavian countries. There has, however, been a substantial increase in the level of this provision over the last ten years, see Ellingsaeter and Hedlund 1998.

2. So-called because they were part of a consortium that cleared all cheques submitted on an overnight basis.

3. The Norwegian bank crisis started in $1987 / 8$ and lasted for approximately seven years (Johansen 1995). The crisis resulted in approximately 10,000 redundancies. By 1 January 1989 the union of bank employees had 32,507 members, but by 1995, this figure was 23,001. In order to avoid bankruptcy, the Norwegian State took over a majority of the shares of the largest Norwegian banks. See Nordhaug and Gooderham et al. 1996; Stene 1992.

4. This raises the difficult problems associated with debates as to the applicability (or otherwise) of 'scientific methods' in sociology. A conclusive resolution of the debates around this issue is not possible and all methods have their limitations. This should not, however, impede efforts to carry out and report on systematic research. See the discussion in Crompton 1999.

5. A full description of the project may be found in Crompton 1999a. The project, and the subsequent research, has been financed by the ESRC and the Norwegian Research Council. Similar interviews have also been carried out in France.

6. Here, however, national differences had to be taken into account. As noted above, the British clearing banks have historically prided themselves on their ability to provide graduate level training, and have recruited school leavers with university level (A level) qualifications, (as well as O- and GCSE-level school leavers). There were therefore fewer graduates amongst the British interviewees. Where appropriate, therefore, some British A-level entrants have been matched with Norwegian graduate entrants.

7. Family circumstances were not matched (although often they turned out to be very similar) as their similarity or variation was one of the factors being examined through the process of case comparison.

8. These numbers relate to the interviews as a whole. It should be noted that the information given in the matching tables, relates to childcare practices at the time of the interview, which in some cases had changed since children were young.

9. It may be noted that in matches 4 and 7 of Table 4, partnerships had foundered/were foundering on account of dissatisfaction with paternal input.

10. In the case of the Norwegian interviews, the job titles are recorded as given. In the British interviews, a grading system was in operation in which the higher the number, the higher 
the level within the management hierarchy. To preserve anonymity, the descriptive labels used do not correspond to those of the actual grading scheme.

11. This point has been ignored by writers such as Blossfeld and Hakim 1997, who would seem to take the status quo - masculine market dominance and in the sexual division of labour in the family - for granted.

12. This serves to make the point that caring might be gender coded, but is not 'gendered' as such.

13. This paper has concentrated on individuals in managerial employment. However, the difficulties faced by those lower down the occupational hierarchy are equally acute and are usefully summarised in Lewis and Lewis, 1996, chapter 12.

\section{REFERENCES}

Blossfeld, H.-P. and Hakim, C. (ed.) (1997) Between Equalization and Marginalization, Oxford: Oxford University Press.

Bock, G. and Thane, P. (eds.) (1991) Maternity and Gender Policies, London: Routledge.

Brandth, B. and Kvande, E. (1999) 'State feminism and flexible fathers', paper given to the to Nordic-UK Seminar, Stockholm, June.

Connell, R. W. (1987) Gender and Power, Cambridge: Polity Press.

Crompton, R. (1989) 'Women in Banking', Work, Employment and Society, 3:141-56.

Crompton, R. (ed.) (1999a) The Restructuring of Gender Relations and Employment, Oxford: Oxford University Press.

Crompton, R. (1999b) 'Comparative Research and Biographical Matching', paper given to the Nordic-UK Seminar, Copenhagen, November.

Crompton, R. and Harris, F. (1997) 'Women's employment and gender attitudes: a comparative analysis of Britain, Norway and the Czech Republic', Acta Sociologica 40: 183-202.

Crompton, R. and Jones, G. (1984) White-Collar Proletariat, London: Macmillan.

Duncan, S. (1995) 'Theorizing European Gender Systems', Journal of European Social Policy, 5 (4): 263-84.

Ellingsaeter, A.-E. and Hedlund, M.-A. (1998) Care resources, employment and gender equality in Norway, ISAF, Oslo.

Ellingsaeter, A.-M. (1999) 'Dual breadwinners between state and market', in Crompton (1999a).

Esping-Andersen, G. (1990) The Three Worlds of Welfare Capitalism, Cambridge: Polity Press.

Halford, S., Savage, M. and Witz, A. (1997) Gender, Careers and Organisations, London: Macmillan.

Hernes, H. (1987) Welfare State and Woman Power, Oslo: Norwegian University Press.

Hochschild, A. (1997) The Time Bind, New York: Metropolitan Books.

Johansen, Fritz P. (1995) En dokumentasjon omkring krisen in finansnæeringen, Hvitbok III. Oslo: Finansforbundet.

Kohn, M. L. (1987) 'Cross-national research as an analytic strategy', American Sociological Review, 52 : 713-31.

Leira, A. (1992) Welfare States and Working Mothers, Cambridge: Cambridge University Press.

Lewis, J. (1992) 'Gender and the Development of Welfare Regimes', Journal of European Social Policy, 2, 3: 159-73.

Lewis, S. and Lewis, J. (1996) The Work-Family Challenge, London: Sage.

Melhuish, M. and Moss, P. (eds.) (1991) Day Care for Young Children, London: Tavistock/Routledge. Mill, J. S. (1961) A System of Logic, London: Longman.

New Ways to Work (1995) Balanced Lives: Changing work patterns for men, London.

Nordhaug, Odd and Gooderham, Paul et al. (1996) Kompetanseutvikling i naringslivet. Oslo: Cappelen. 
O’Reilly, J. and Fagan, C. (eds.) (1998) Part-time Prospects, London: Routledge.

OECD (1994) Women and Structural Change, Paris.

Pfau-Effinger, B. (1993) 'Modernisation, Culture and Part-time Employment', Work, Employment and Society, 7: 383-410.

Pickvance, C. (1995) 'Comparative analysis, causality and case studies in Urban Studies', in A. Rogers and S. Vertovec, The Urban Context, Oxford and Washington: Berg.

Ragin, C. (1987) The Comparative Method, Berkeley and Los Angeles: University of California Press.

Ragin, C. (1991) (ed.) 'Introduction' to Issues and Alternatives in Comparative Social Research, Leiden: E. J. Brill.

Ragin, C. and Zaret, D. (1983) 'Theory and Method in Comparative Research', Social Forces, 731-54.

Rubery, J., Smith, M., Fagan, C. and Grimshaw, D. (1998) Women and European Employment, London: Routledge.

Sainsbury, D. (ed.) (1994) Gendering Welfare States, London: Sage.

Skocpol, T. and Somers, M. (1980) 'The uses of comparative history in macrosocial inquiry', Comparative Studies in Society and History, 22, 2: 174-97.

Stene, E. M. (1992) Bank i bordet! Strukturendringer i banknæringen og omstillingsprosesser for de ansatte. Oslo: Fafo-report no. 141.

Rosemary Crompton

Department of Sociology

School of Social \& Human Sciences

City University

Northampton Square

LONDON EC1V oHB

Gunn Birkelund

Department of Sociology

University of Oslo

Accepted December 1999

NORWAY 